Tohoku J. Exp. Med., 2003, 199, 197-203

\title{
Urethral Recurrence Following Neobladder in Bladder Cancer Patients
}

\author{
Shinichi Yamashita, Senji Hoshi, Chikara Ohyama, Makoto Satoh, \\ Fuminiko Soma, Yasuhiro Okada, Shinnosuke Kato, Ichiro Shintaku and \\ Yoichi ARAi \\ Department of Urology, Tohoku University School of Medicine, Sendai 980- \\ 8574
}

Yamashita, S., Hoshi, S., Ohyama, C., Satoh, M., Soma, F., Okada, Y., Kato, S., Shintaku, I. and ARAI, Y. Urethral Recurrence Following Neobladder in Bladder Cancer Patients. Tohoku J. Exp. Med., 2003, 199 (4), 197-203 — Risk factors of urethral recurrence after neobladder in bladder cancer patients were studied. Between 1977 and 2001, 73 patients (male 58, female 15) underwent neobladder as a treatment for bladder cancer. The observation time after cystectomy ranged from 2 to 254 months (median 60.5). Ten (17.2\%) of 58 male patients had urethral recurrence and of the 10, 8 patients had multiple bladder cancers including bladder neck. Urethral recurrence was found by macrohematuria, follow-up cystourethroscopy, and inguinal lymph node swelling. Only one who complained of macrohematuria had positive urinary cytology. Of 58 male patients, 5 underwent total nephroureterectomy for renal pelvic or ureteral cancer before radical cystectomy, and 3 of the 5 had urethral recurrence. Two of 10 patients with urethral recurrence died with cancer, and they had renal pelvic or ureteral cancer. The five-year cause specific survival was $83 \%$ for patients with urethral recurrence, and 79\% for those without urethral recurrence, respectively. Urethral recurrence did not have a significant effect on survival. The patients with multiple bladder cancers including bladder neck, and renal pelvic or ureteral cancer before radical cystectomy, have high risks for urethral recurrence. Urinary cytology has limited value for the detection of urethral recurrence. urethral recurrence; bladder cancer; neobladder

(C) 2003 Tohoku University Medical Press

The risk of urethral recurrence after radical cystectomy for bladder cancer has been well documented. It has been reported that significant risk factors in bladder cancer related to the development of cancer in the retained urethra were papillary cancer, multiple cancers, and tumors in the bladder neck, prostatic urethra and prostatic gland (Tobisu et al. 1991).

Multiple development of transitional cell carcinoma in the entire urinary tract, that is

Received April 1, 2002; revision accepted for publication April 2, 2003.

Address for reprints: Senji Hoshi, M.D., Department of Urology, Yamagata Prefectural Central Hospital,

1800 Aoyagi, Yamagata 990-2292, Japan.

e-mail: hoshi@ uro.med.tohoku.ac.jp 
renal pelvis, ureter, bladder and urethra, has been well documented (Kakizoe et al. 1991). It has been reported that the incidence of transitional cell carcinoma in the bladder after total nephroureterectomy for transitional cell carcinoma of the renal pelvis and/or ureter ranges from 15 to 50\% (Newman et al. 1967; Williams and Mitchell 1970). The incidence of urethral recurrence after radical cystectomy for bladder cancer has been reported to range from 0.7 to 18\% (Freeman et al. 1994).

We report risk factors of urethral recurrence after neobladder for bladder cancer.

\section{PATIENTS AND Methods}

Between July 1977 and June 2001, 73 patients, including 58 males and 15 females, underwent radical cystectomy and neobladder as a treatment for bladder cancer at our institution. Since no female patient had urethral recurrence, we excluded female patients from this study. The ages of the male patient ranged from 40 to 74 years old (median age 64 years). Of the 58 male patients, 5 underwent total nephroureterectomy for renal pelvic or ureteral cancer before radical cystectomy.

One to six courses of chemotherapy with 70 $\mathrm{mg} / \mathrm{m}^{2}$ cisplatin, $30 \mathrm{mg} / \mathrm{m}^{2}$ methotrexate, and $30 \mathrm{mg} / \mathrm{m}^{2}$ THP-adriamycin were administrated to 38 patients as neoadjuvant therapy. Seven patients were treated with chemotherapy combined with $40 \mathrm{~Gy}$. irradiation to the pelvic space.

The TNM classification was used for clinical staging, diagnosed by transurethral resection (TUR) biopsy, pyelography, computerized tomo- graphy (CT), magnetic resonance imaging (MRI), whole-layer core biopsy (WLCB) of the bladder tumor (Hoshi et al. 2001), and percutaneous fine needle aspiration biopsy (FNAB) of regional lymphoid nodes (Hoshi et al. 1999). The patterns of bladder tumor were analyzed by pathological stage (pT), grade $(\mathrm{G})$ and location in the cystectomy specimens. Pathological stage and grade of the 58 patients are shown in Table 1.

The observed period after radical cystectomy for bladder cancer ranged from 2 to 254 months (median 60.5 months). Urinary cytology and cystourethroscopy were performed as follow-up. Urethral recurrence was pathologically diagnosed by the biopsy or transurethral resection of the urethral tumor.

A $\chi^{2}$-test was used to assess the statistical significance of differences. Cause-specific survival and urethral recurrence rate was calculated by the Kaplan-Meier method. For evaluation of the survival rate, the starting point was designated as the day of radical cystectomy and end point as the last day of follow-up. Logrank test was used to assess differences of the survival rate and the differences with a $p<0.05$ were considered significant.

\section{RESULTS}

Ten $(17.2 \%)$ of the 58 male patients had urethral recurrence (Table 2). The ages of the patients with urethral recurrence ranged from 55 to 73 years old (median age 66.5 years), while those without urethral recurrence ranged from

\section{TABle 1. Pathological stage ( $(D T)$ and grade $(G)$ in cystectomy specimens}

\begin{tabular}{|c|c|c|c|c|c|c|c|c|}
\hline & \multicolumn{8}{|c|}{ No. patients with urethral recurrence } \\
\hline & pT0 & pTis & $\mathrm{pTa}$ & $\mathrm{pT} 1$ & pT2 & pT3 & pT4 & Total \\
\hline & 8 & & & & & & & 8 \\
\hline G1 & & & 1 & 2 & & & & 3 \\
\hline G2 & & 6 & $2(1)$ & $8(3)$ & 1 & 1 & 2 & $20(4)$ \\
\hline G3 & & $4(2)$ & $2(1)$ & $8(1)$ & $7(1)$ & 4 & $2(1)$ & $27(6)$ \\
\hline Total & 8 & $10(2)$ & $5(2)$ & $18(4)$ & $8(1)$ & 5 & $4(2)$ & $58(10)$ \\
\hline
\end{tabular}


TABLE 2. Cases with urethral recurrence

\begin{tabular}{|c|c|c|c|c|c|c|c|}
\hline Case & Age & $\mathrm{pT} / \mathrm{G}$ & $\begin{array}{l}\text { Past } \\
\text { history }\end{array}$ & $\begin{array}{l}\text { Interval }^{1} \\
\text { (months) }^{2}\end{array}$ & $\begin{array}{l}\text { Site of } \\
\text { urethral rec. }\end{array}$ & $\mathrm{G}^{2}$ & Status \\
\hline 1 & 72 & Tis/G3 & & 6 & Anterior & G2 & Dead with another cause \\
\hline 2 & 55 & T2/G3 & & 11 & Anterior & G2 & Alive \\
\hline 3 & 57 & $\mathrm{Ta} / \mathrm{G} 2$ & Renal pelvic tumor & 11 & Anterior & $\mathrm{G} 2$ & Dead with cancer \\
\hline 4 & 69 & Tis/G3 & Renal pelvic tumtr & 18 & Anastomosis & G3 & Alive \\
\hline 5 & 73 & $\mathrm{~T} 1 / \mathrm{G} 2$ & & 21 & Anterior & G2 & Alive \\
\hline 6 & 67 & $\mathrm{~T} 1 / \mathrm{G} 2$ & & 22 & Posterior & G2 & Dead with another cause \\
\hline 7 & 65 & T4/G3 & & 27 & Anastomosis & G3 & Alive \\
\hline 8 & 59 & $\mathrm{~T} 1 / \mathrm{G} 2$ & Ureteral tumor & 29 & Anterior & G2 & Dead with cancer \\
\hline 9 & 66 & $\mathrm{~T} 1 / \mathrm{G} 3$ & & 50 & Anterior & G2 & Alive \\
\hline 10 & 68 & $\mathrm{Ta} / \mathrm{G} 3$ & & 100 & Anastomosis & G2 & Dead with another cause \\
\hline
\end{tabular}

${ }^{1}$ Interval between cystectomy and urethral recurrence.

${ }^{2}$ Grade of urethral recurrence.

40 to 74 years old (median age 62 years). The pathological stage (pT) and grade $(\mathrm{G})$ in the cystectomy specimens of 10 patients with urethral recurrence was pTis in 2 patients, $\mathrm{pTa}$ in 2 , pT1 in 4, pT2 in 1, pT4 in 1, G1 in 1, G2 in 4, and G3 in 5 (Table 1).

The durations between cystectomy and detection of urethral recurrence ranged from 6 to 100 months (median 21.5). The five-, tenand twenty-year urethral recurrence rate were $21 \%, 29 \%$ and $29 \%$, respectively. Of the 10 patients with urethral recurrence, 8 patients had multiple bladder cancers including bladder neck. All of 8 had urethral recurrence within 29 months (Table 2, Case 1 8). One had invased the prostate with concomitant carcinoma-in-situ (CIS). Eight (27.6\%) of 29 patients with bladder neck involvement had urethral recurrence, but only two $(6.9 \%)$ of 29 patients without bladder neck involvement had urethral recurrence. The bladder neck involvement had significant effect on urethral recurrence $(\phi=0.039)$.

Urethral recurrence was found by macrohematuria in 3 patients, by follow-up cystourethroscopy in 6 , and by inguinal lymph node swelling in 1. Bladder cancer rarely transferred to inguinal lymph node. Then urethal recurrence was found when urethroscopy was perfomed. Only one who complained of macrohematuria had positive urinary cytology. The sites of urethral recurrence were the anterior urethra in 6 patients, the posterior urethra in 1 and the anastomosis in 3. Urethral recurrence was pathologically diagnosed as transitional cell carcinoma and the grades are shown in Table 2.

The observation time after urethral recurrence ranged from 3 to 90 months (median 18.5). The clinical courses after urethral recurrence are shown in Fig. 1. All patients were treated by TUR and chemotherapy with or without irradiation. TUR was performed from once to twenty times and after those treatments, the patients were able to void spontaneously. Five of 10 patients remain alive, while 2 died of cancer and 3 others died of other diseases (cardiac infarction, renal failure, and suicide). The intervals between urethral recurrence and radical cystectomy, and the survival time after urethral recurrence are shown in Fig. 2. Although the interval was not clearly related to the survival, 2 patients who died of cancer underwent nephroureterectomy for renal pelvic or ureteral cancer prior to radical cystectomy.

Of 58 male patients, 5 underwent total nephroureterectomy for renal pelvic or ureteral cancer from 9 to 108 months (median 72) before radical cystectomy. The five patients are listed in Table 3. Three $(p=0.039)$ of the 5 had 


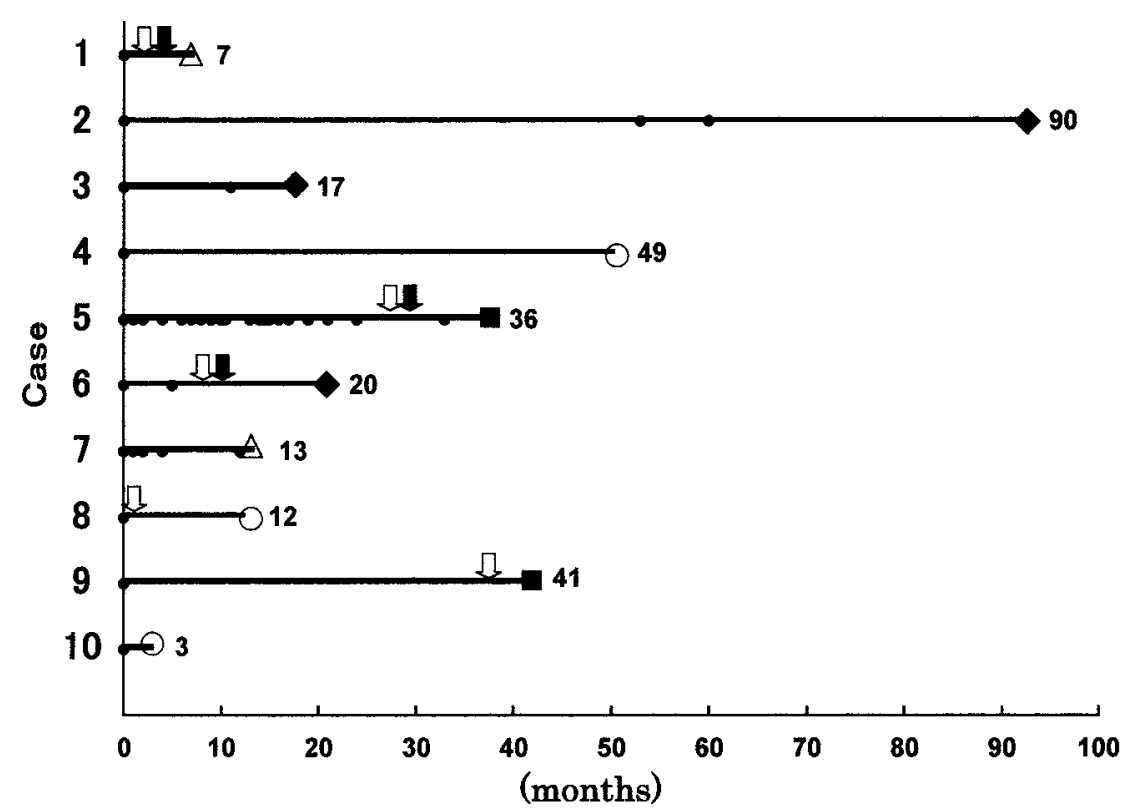

Fig. 1. Clinical course after urethral recurrence.

- uretheral recurrence; $\bigcirc$, no evidence of disease; $\triangle$, alive with disease; $\mathbf{\square}$, dead with cancer; $\boldsymbol{\nabla}$, dead with another disease. Therapy after urethral recurrence, $\Rightarrow$, chemotherapy; $\rightarrow$, radiation.

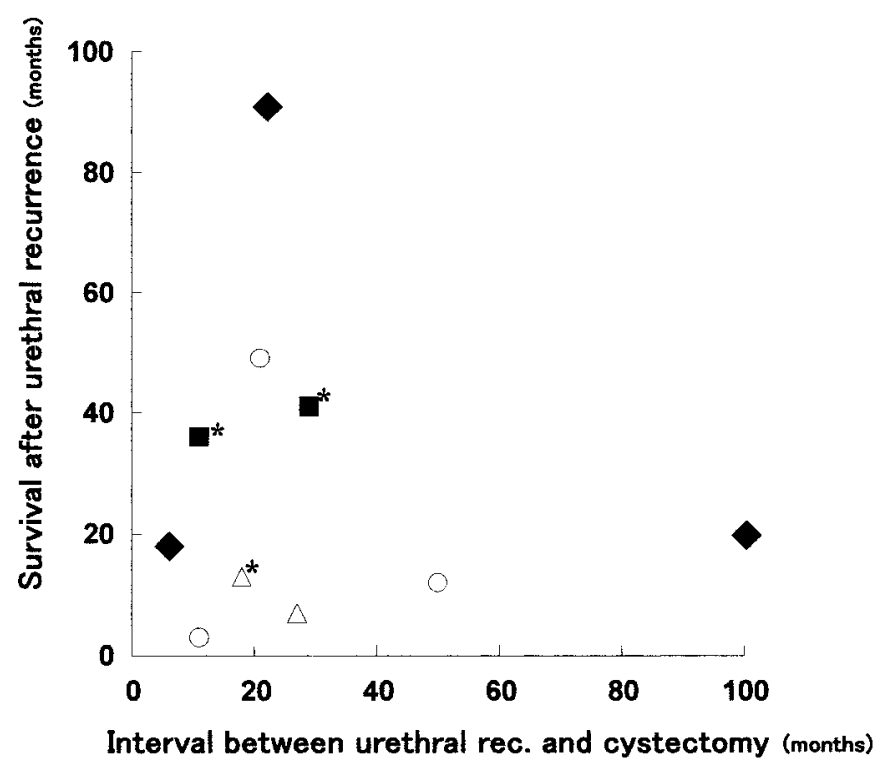

Fig. 2. Survival after urethral recurrence.

$\bigcirc$, no evidence of disease; $\triangle$, alive with disease; disease. * with renal pelvic or ureteral cancer.

urethral recurrence, compared with patients who had not have renal pelvic or ureteral cancer.

The five-year cause specific, and over all survival rates for the patients with urethral recurrence were $83 \%$ and $74 \%$, and $79 \%$ and $75 \%$ for those without urethral recurrence, respectively. The cause specific survival was shown in Fig. 3. Urethral recurrence did not have a significant effect on survival $(p=0.983)$. 
TABLE 3. Cases with renal pelvic or ureteral cancer prior to radical cystectomy

\begin{tabular}{ccllcccl}
\hline Case & Age & $\begin{array}{c}\text { Site of renal pelvic/ } \\
\text { ureteral tumor }\end{array}$ & pT/G & Interval $^{1}$ & $\begin{array}{c}\text { Observed } \\
\text { time }^{2}\end{array}$ & $\begin{array}{c}\text { Urethral } \\
\text { rec. }\end{array}$ & Status \\
\hline 3 & 57 & Renal pelvis & pT1/G2 & 9 & 47 & & Dead with cancer \\
8 & 59 & Lower ureter & pT2/G2 & 72 & 70 & & Dead with cancer \\
4 & 69 & Renal pelvis & pT1/G3 & 80 & 31 & Alive \\
11 & 62 & Lower ureter & pT2/G2 & 30 & 57 & No & Alive \\
12 & 60 & Renal pelvis & Unknown & 108 & 5 & No & Alive \\
\hline
\end{tabular}

${ }^{1}$ Interval between nephroureterectomy and cystectomy (months).

${ }^{2}$ Ovserved time from cystectomy (months).

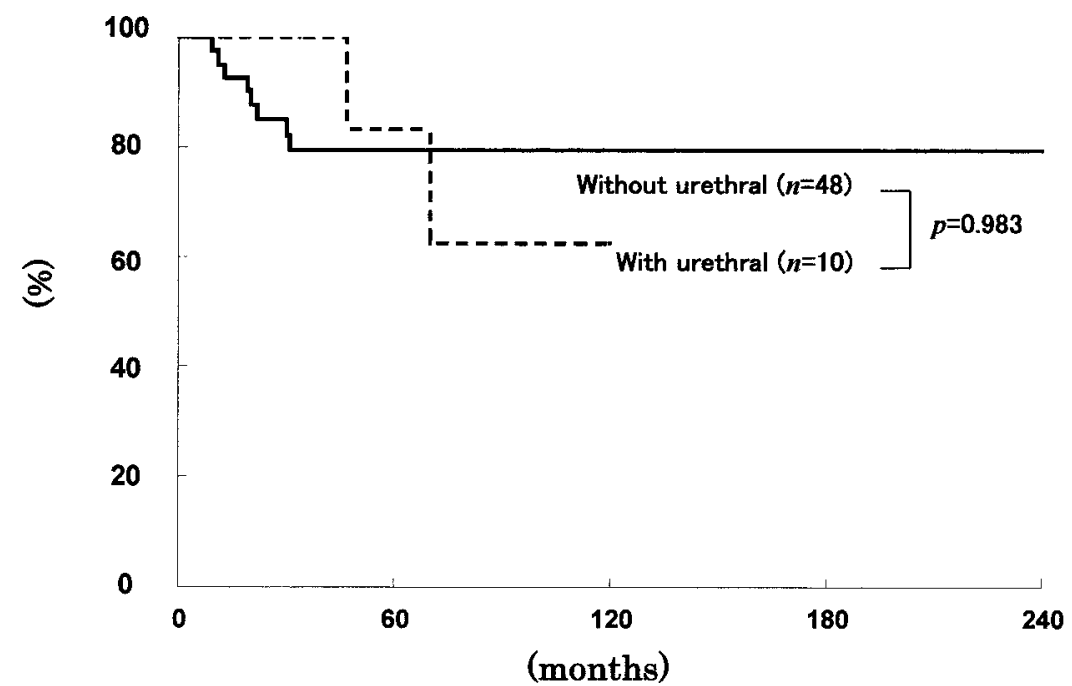

Fig. 3. Cause specific survival rate with/without urethral recurrence. Urethral recurrence did not have a significant effect on survival.

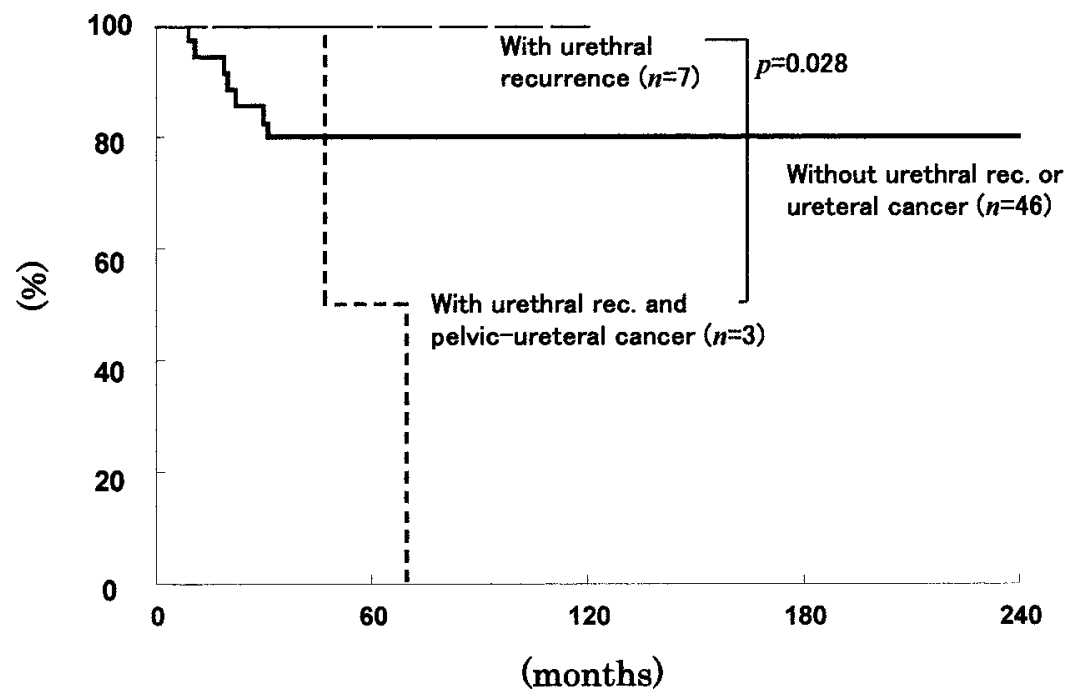

Fig. 4. Cause specific survival rate with/without renal pelvic or ureteral cancer. With urethral recurrence and renal pelvic-ureteral cancer, the survival was significantly poor. Except 2 patients (Table 3, Case 11,12) without urethral recurrence and with renal pelvic-ureteral cancer. 
Fig. 4 shows the five-year cause specific survival of the patients who underwent radical cystectomy following to nephroureterectomy and had urethral recurrence. The five-year survival was $50 \%$, and significantly poor $(p=$ 0.028).

\section{DISCUSSION}

The risk of urethral recurrence after radical cystectomy for bladder cancer has been well documented. The incidence of urethral recurrence after radical cystectomy for bladder cancer has been reported to range from 0.7 to $18 \%$ (Freeman et al. 1994). Recent reports have suggested a lower rate of urethral recurrence after cystectomy with orthotopic reconstruction. Freeman et al. (1996) have reported that patients with ileal neobladder had a significantly lower probability of urethral recurrence (2. 9\%) compared with patients with cutaneous diversion (11.1\%).

Urethral recurrence, however, occurred in ten $(17.2 \%)$ of the 58 male patients who had undergone radical cystectomy and neobladder as a treatment for bladder cancer at our institution between July 1977 and June 2001. Tobisu et al. (1991) has reported that significant risk factors in bladder cancer related to the development of cancer in the retained urethra were multiple cancers, and tumors in the bladder neck, prostatic urethra and prostatic gland. Of our 10 patients with urethral recurrence, 8 patients had multiple bladder cancers including bladder neck involvement. Accordingly bladder neck involvement may be a significant risk factor for urethral recurrence after cystectomy. If the patients, who had multiple bladder cancers including bladder neck, did not undergo radical cystectomy and neobladder as a treatment for bladder cancer, the rate of urethral recurrence might have been reduced.

Generally, the duration between cystectomy and detection of urethral recurrence has mainly been within 5 years (Tobisu et al.
1991). In this study, the durations ranged from 6 to 100 months (median 21.5). In 8 patients, urethral recurrence was found within 29 months after cystectomy, but, in 2 other patients, the durations were 50 and 100 months, respectively. Therefore, long term follow-up is important for detection of urethral recurrence.

Six of 10 patients with urethral recurrence were found by follow-up cystourethroscopy. Three of 10 patients with urethral recurrence complained of macrohematuria, and only one of them had positive urinary cytology. With regard to the detection of early urethral recurrence after radical cystectomy, the follow-up cystourethroscopy was more useful than urinary cytology.

Multiple development of transitional cell carcinoma in the urinary tract was well recognized (Kakizoe et al. 1991). Three of 10 patients with urethral recurrence in this study underwent nephroureterectomy prior to radical cystectomy. In 58 male patients, 5 underwent total nephroureterectomy for renal pelvic or ureteral cancer before radical cystectomy. Three $(p=0.039)$ of 5 had urethral recurrence. The survival was significantly poor $(p=0.028)$. The patients with bladder cancer following renal pelvic or ureteral cancer had better avoid neobladder simultaneously because of high risk for urethral recurrence. If the patient with renal pelvic or ureteral cancer requested neobladder after informed consent, the follow-up cystourethroscopy should be performed frequently. Machele Donat et al. (2001) reported that prostatic involvement was translated into a higher risk of urethral recurrence, however, it did not have significant clinical impact or affect survival. In this study, the urethral recurrence did not have significant effect on survival $(p=0$. 983). All our patients with urethral recurrence were treated by TUR and chemotherapy with or without irradiation. After those treatments, they were able to void spontaneously.

Our study shows that the patients who have multiple bladder cancers including bladder neck, 
and renal pelvic or ureteral cancer before radical cystectomy have high risk for urethral recurrence after neobladder. Urinary cytology has limited value for the detection of urethral recurrence. After 100 months, one patient still developed urethral recurrence. We, therefore, recommend the follow-up cystourethroscopy.

\section{References}

Freeman, J.A., Esrig, D. \& Stein, J.P. (1994) Management of the patient with bladder cancer. Urethral recurrence. Urol. Clin. North Am., 21, 645-651.

Freeman, J.A., Tarter, T.A. \& Esrig, D. (1996) Urethral recurrence in patients with orthotopic ileal neobladder. J. Urol., 156, 1615 -1619 .

Hoshi, S., Orikasa, S. \& Yoshikawa, K. (1999) Neoadjuvant therapy and bladder substitute for invasive bladder cancer: 20 years experience at Tohoku University. Int. J. Urol., 6, 68-74.

Hoshi, S., Orikasa, S. \& Ono, K. (2001) Trans- urethral whole layer core biopsy for detection of residual tumor after neoadjuvant therapy invasive bladder cancer. Urol. Oncol., 6, 85 $-89$

Kakizoe, T., Tobisu, K. \& Tanaka, Y. (1991) Development of multiple transitional cell carcinoma in the urinary tract. Jpn. J. Clin. Oncol., 21, $110-114$.

Machele Donat, S., David, C. \& Michael, S. (2001) The efficacy of transurethral biopsy for predicting the long-term clinical impact of prostatic invasive bladder cancer. J. Urol., 165, 1580-1584.

Newman, D.M., Allen, L.E. \& Wishard, W.N. (1967) Transitional cell carcinoma of the upper tract. J. Urol., 98, 322-327.

Tobisu, K., Tanaka, Y. \& Mizutani, T. (1991) Transitional cell carcinoma of the urethra in men following cystectomy for bladder cancer: multivariate analysis for risk factors. $J$. Urol., 146, 1551-1553.

Williams, C.B. \& Mitchell, J.P. (1970) Carcinoma of the renal pelvis. A review of 43 cases. Br. J. Urol., 45, 370-376. 